\title{
Corrigendum: NCNet: Deep Learning Network Models for Predicting Function of Non-Coding DNA
}

\section{OPEN ACCESS}

Approved by:

Frontiers Editorial Office,

Frontiers Media SA,

Switzerland

${ }^{*}$ Correspondence:

Che-Lun Hung

clhung@mail.cgu.edu.tw

Specialty section:

This article was submitted to

Bioinformatics and

Computational Biology,

a section of the journal

Frontiers in Genetics

Received: 03 September 2019 Accepted: 04 September 2019 Published: 13 September 2019

Citation:

Zhang H, Hung C-L, Liu M, Hu X and Lin Y-Y (2019) Corrigendum: NCNet:

Deep Learning Network Models for

Predicting Function of

Non-Coding DNA

Front. Genet. 10:923.

doi: 10.3389/fgene.2019.00923

\begin{abstract}
Hanyu Zhang ${ }^{1,2}$, Che-Lun Hung ${ }^{3,4,5,6 *}$, Meiyuan Liu ${ }^{7}$, Xiaoye Hu ${ }^{7}$ and Yi-Yang Lin ${ }^{6}$
${ }^{1}$ College of Computing and Informatics, Providence University, Taichung City, Taiwan, ${ }^{2}$ Labo MICS, École CentraleSup élec, Université Paris Saclay, Gif-sur-Yvette, France, ${ }^{3}$ Department and Graduate Institute of Computer Science and Information Engineering, Chang Gung University, Taoyuan City, Taiwan, ${ }^{4}$ Division of Rheumatology, Allergy and Immunology, Chang Gung Memorial Hospital, Taoyuan City, Taiwan, ${ }^{5}$ Al Innovation Research Center, Chang Gung University, Taoyuan City, Taiwan,

${ }^{6}$ Department of Computer Science and Communication Engineering, Providence University, Taichung City, Taiwan,

${ }^{7}$ Affiliated Cancer Hospital \& Institute of Guangzhou Medical University, Guangzhou, China
\end{abstract}

Keywords: Non-coding DNA, residual learning, LSTM, sequence to sequence learning, deep learning

\section{A corrigendum on}

NCNet: Deep Learning Network Models for Predicting Function of Non-Coding DNA

by Zhang H, Hung C-L, Liu M, Hu X and Lin Y-Y (2019). Front. Genet. 10:432. doi: 10.3389/ fgene.2019.00432

In the published article, there was an error in affiliation "1." The affiliation "Affiliated Cancer Hospital \& Institute of Guangzhou Medical University, Guangzhou, China" should be moved to affiliation "7" and should be removed for the first and corresponding authors. Additionally, all subsequent affiliations should move up in order.

The authors apologize for this error and state that this does not change the scientific conclusions of the article in any way. The original article has been updated.

Copyright (c) 2019 Zhang, Hung, Liu, Hu and Lin. This is an open-access article distributed under the terms of the Creative Commons Attribution License (CC BY). The use, distribution or reproduction in other forums is permitted, provided the original author(s) and the copyright owner are credited and that the original publication in his journal is cited, in accordance with accepted academic practice. No use, distribution or reproduction is permitted which does not comply with these terms. 